\title{
Alpha-tocopherol content in 62 edible tropical plants
}

\begin{abstract}
Vitamin E was determined by the high-performance liquid chromatography (HPLC) method. All the plants tested showed differences in their Ŭ-tocopherol content and the differences were significant $(\mathrm{p}<0.05)$. The highest $\breve{U}$ tocopherol content was in Sauropus androgynus leaves $(426.8 \mathrm{mg} / \mathrm{kg}$ edible portion), followed by Citrus hystrix leaves $(398.3 \mathrm{mg} / \mathrm{kg}$ ), Calamus scipronum (193.8 mg/kg), starfruit leaves Averrhoa belimbi $(168.3 \mathrm{mg} / \mathrm{kg})$, red pepper Capsicum annum $(155.4 \mathrm{mg} / \mathrm{kg})$, local celery Apium graveolens $(136.4 \mathrm{mg} / \mathrm{kg})$, sweet potato shoots Ipomoea batatas $(130.1 \mathrm{mg} / \mathrm{kg})$, Pandanus odorus $(131.5 \mathrm{mg} / \mathrm{kg})$, Oenanthe javanica $(146.8 \mathrm{mg} / \mathrm{kg})$, black tea Camelia chinensis $(183.3 \mathrm{mg} / \mathrm{kg})$, papaya Carica papaya shoots $(111.3 \mathrm{mg} / \mathrm{kg})$, wolfberry leaves Lycium chinense $(94.4 \mathrm{mg} / \mathrm{kg})$, bird chili Capsicum frutescens leaves $(95.4 \mathrm{mg} / \mathrm{kg})$, drumstick Moringa oleifera leaves $(90.0 \mathrm{mg} / \mathrm{kg})$, green chili Capsicum annum $(87 \mathrm{mg} / \mathrm{kg})$, Allium fistulosum leaves $(74.6 \mathrm{mg} / \mathrm{kg})$, and bell pepper Capsicum annum $(71.0 \mathrm{mg} / \mathrm{kg})$. ǓTocopherol was not detected in Brassica oleracea, Phaeomeria speciosa, Pachyrrhizus speciosa, Pleurotus sajor-caju, and Solanum melongena.
\end{abstract}

Keyword: Edible tropical plants; Ŭ-tocopherol; Vitamin E 